\title{
COSMIC RAYS AND THE DYNAMIC BALANCE IN THE LARGE MAGELLANIC CLOUD
}

\author{
CARL E. FICHTEL \\ Goddard Space Flight Center, Greenbelt, Maryland \\ MEHMET E. OZEL \\ Çukurova \\ and \\ ROBERT G. STONE \\ Goddard Space Flight Center, Greenbelt, Maryland
}

\begin{abstract}
Present and future measurement of the Large Magellanic Cloud (LMC) particularly in the radio and high energy gamma ray range offer the possibility of understanding the density and distribution of the cosmic rays in a galaxy other than our own and the role that they play in galactic dynamic balance. After a study of the consistency of the measurements and interpretation of the synchrotron radiation from our own galaxy, the cosmic ray distribution for the LMC is calculated under the assumption that the cosmic ray nucleon to electron ratio is the same and the relation to the magnetic fields are the same, although the implications of alternatives are discussed. It is seen that the cosmic ray density level appears to be similar to that in our own galaxy, but varying in position in a manner generally consistent with the concept of correlation with the matter on a broad scale.
\end{abstract}

\section{Introduction}

Through observations at many frequencies including the radio and the high energy gamma ray region, studies of the Large Magellanic Cloud (LMC) offer the possibilities of learning about the nature and role of cosmic rays in a galaxy other than our own. Although the greater distance is a significant handicap for some of the measurements, there is the clear advantage of being able to look at the galaxy from the outside rather than looking at the superposition of the radiation from several arms as in the case of our galaxy. The combination of the $21 \mathrm{~cm}$ and CO radio measurements and future medium and high energy gamma ray measurements provide information on the density and energy spectrum of the cosmic ray nucleons and at the same time a better normalization for the molecular hydrogen density estimates deduced from the CO measurements (Ozel and Fichtel, 1988). The radio continuum synchrotron observations provide information on the cosmic-ray electron spectrum as a function of position in the galaxy. The understanding of the latter is complicated by the lack of knowledge of the magnetic field strength and distribution and by some uncertainty in the interpretation of the synchrotron radiation in our own galaxy. There are, nonetheless, reasonable consistency and similarity arguments which can be used to generate a self consistent model of the cosmic rays, magnetic fields, and matter.

Y. Kondo (ed.), Observatories in Earth Orbit and Beyond, 537-541.

(C) 1990 Kluwer Academic Publishers. Printed in The Netherlands. 


\section{Cosmic Rays, Matter, Magnetic Fields, and Dynamic Balance}

In order to attempt to understand the matters relevant to cosmic rays in the LMC, it is valuable to look first at the situation in our own galaxy and to remember that the bulk of the energy is in the cosmic ray nucleons (by a factor of about $10^{2}$ ) and not the cosmic ray electrons, which produce the synchrotron radiation. In our galaxy, the local properties of the cosmic rays are well known (e.g., Simpson, 1983, Webber, 1983), but the nature of the solar modulation is not. The cosmic rays are depressed in intensity in an energy dependent manner in the vicinity of the Sun by a combination of effects including convection, diffusion, and energy changes due to the expansion of the solar wind. The exact nature of the energy dependence is not well understood, in spite of considerable theoretical effort (e.g., Parker, 1965; Gleason and Axford, 1968; Fisk, 1971). There does, however, seem to be agreement, based to a large degree on the observation of the changes in the cosmic ray intensity as a function over the solar cycle, that the degree of modulation decreases markedly with increasing energy and is quite small above about $10^{2} \mathrm{GeV}$. This last deduction together with the galactic synchroton and gamma ray measurements was used by Fichtel, Stone, and Ozel (1990) to reconstruct an interstellar electron spectrum. The relationship between the electron spectrum in our galaxy and that in the LMC can be deduced from the synchrotron measurements. The solar modulation effect on the nucleonic component is less severe because of the much larger particle mass.

The matter density in the LMC, or at least the column density, is a much more straight-forward subject to discuss. The atomic hydrogen density may, of course, be obtained directly from the 21 centimeter data. The 21 centimeter map of the LMC given by Mathewson and Ford (1984) is used here for the reasons given in the paper by Ozel and Fichtel (1988). The heavier nuclei will be assumed to be present in the same relatively small abundances that they are in our galaxy. For the molecular hydrogen density, the CO map may be used to provide the distribution, although the normalization is uncertain. The recently completed full survey of the central $6 \mathrm{deg} \times 6 \mathrm{deg}$ by Cohen et al. (1988) will be taken for this purpose. The normalization factor for the $\mathrm{N}_{\mathrm{H}_{2}} / \mathrm{W}_{\mathrm{CO}}$ ratio of $1.7 \times 10^{21} \mathrm{~cm}^{-2} \mathrm{~K} \mathrm{~km} \mathrm{~s}^{-1}$ will be used here (Cohen et al., 1988). As Ozel and Fichtel note, since the atomic and molecular hydrogen density distributions are quite different, future high energy gamma ray measurements may help to improvement the normalization value.

\section{Synchroton Radiation}

If the electrons have a spectrum of the form

$$
N(E)=K E^{-\gamma} d E
$$

where $N(E)$ is the number of electrons erg s $\mathrm{s}^{-1} \mathrm{~cm}^{-3}$, and where the electrons are homogenous and isotropic, then Ginzburg and Syrovatskii (1964) have shown that the intensity of the radiation is given by

$$
I_{\nu}=1.35 \times 10^{-22} a(\gamma) \mathrm{LKH}^{(\gamma+1) / 2}\left(\frac{6.26 \times 10^{18}}{\nu}\right)^{(\gamma-1 / 2)} \operatorname{ergs~cm}^{-2} \mathrm{~s}^{-1} \mathrm{ster}^{-1} \mathrm{~Hz}^{-1}
$$


in the presence of random magnetic fields. In this expression, $a$ is a slowly varying function of $q$ with a value near 0.1 for the range of interest here, $L$ is the length over which the electrons and magnetic fields are present, and $H$ is the magnetic field strength. From equation (2), it is seen that, if the synchrotron spectrum is known, giving $I$ as a function of over a reasonable frequency range, and $L$ can be estimated, then $K$ may be determined if $H$ is known.

It is also important to know the relationship between the maximum in tne synchrotron radiation and a given electron energy, which is (Webber, 1983).

$$
E_{\text {eff }}=2.5 \times 10^{2}\left(\frac{\nu}{H_{\perp}}\right)^{1 / 2} \mathrm{eV}
$$

The frequency range of interest in the LMC study here is from about 20 to 1400 $\mathrm{MHz}$, corresponding then to electrons in the energy range from approximately 0.50 to $4.2 \mathrm{GeV}$ for a $5 \mu \mathrm{G}$ field, or in fact a somewhat broader range when the distribution functions are considered, and 0.30 to $2.5 \mathrm{GeV}$ for a $14 \mu \mathrm{G}$ field.

It should be mentioned at this point, that historically there had been a concern regarding the synchrotron radiation observed in our galaxy. This was that the level appeared to be higher than would have been expected on the basis of the deduced electron spectrum and the magnetic field thought to exist. Recently, however, Fichtel, Ozel, and Stone (1990) have shown that with the interstellar cosmic ray electron spectrum now believed to exist based in part on the SAS-2 and COS-B gamma ray data at high latitudes, and the range of values for the total magnetic field including the random part now estimated from several sources, agreement can be obtained. These authors deduce a random magnetic field of about $11 \mu \mathrm{G}$ for the local region, consistent with the currently estimated range of 5 to $14 \mu \mathrm{G}$, deduced in other ways.

\section{The Large Magellanic Cloud}

Fichtel, Ozel, and Stone further show that within the uncertainties of existing data the nonthermal radiation from the LMC and our galaxy have the same spectral shape. They then assume that the magnetic field pressure density in the LMC has the same relationship to the cosmic ray pressure as that in our galaxy as well as the relationship between the cosmic ray energy density and the electron spectrum being the same in the LMC as in our galaxy. Since the ratios of the cosmic ray electron intensity to the cosmic ray nucleon density and that to the magnetic field are assumed to be the same as in our galaxy, there is a fixed relation between $K$ and $H$ in Equation (2). Specifically, since the magnetic field pressure is proportional to $H_{2}$ if $K_{0}$ and $H_{0}$ are the local values of $K$ and $H$ in our galaxy and $w\left(x_{i}\right) K_{0}$ is a value in a local region of the LMC, then the corresponding $H$ value in the LMC is $\left[w\left(x_{i}\right)\right]^{0.5} H_{0}$ to maintain the relationship between the cosmic-ray and magnetic fields described earlier.

If $L$ is known, $w\left(x_{i}\right)$ may be determined from the knowledge of the synchrotron radiation. Following Klein et al. (1989), Fichtel, Ozel, and Stone used an effective disc thickness for $L$ of $1 \mathrm{kpc}$, the same as in our galaxy. (Remember $L$ for the LMC is the full thickness, not the half thickness as in our galaxy where the Sun is in the 


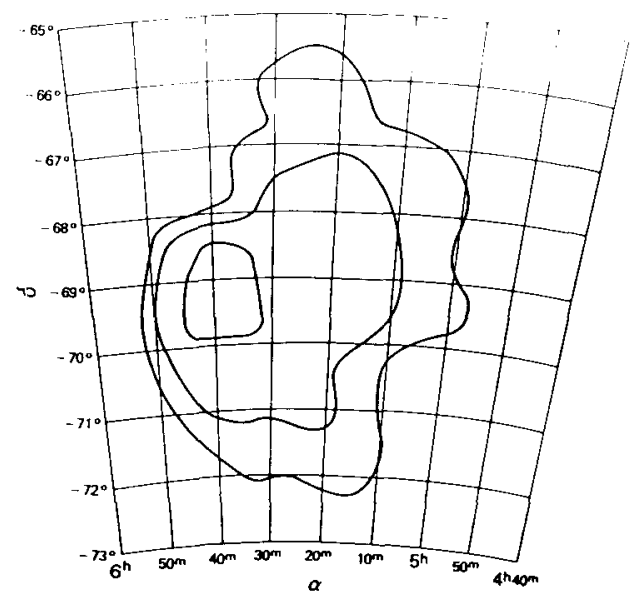

Fig. 1. The contour levels for the predicted cosmic ray energy density levels relative to those in our galaxy based on the $1400 \mathrm{MHz}$ data of Haynes et al. (1986). The contour levels shown et al. (1986). The contour correspond to $w$ equal to $0.59,0.78$, and 1.01 . The contents of the figure are from Fichtel, Ozel, and Stone (1990).

middle of the plane.) They then proceed to calculate was a function of position for the LMC.

They studied three frequencies $45 \mathrm{MHz}, 408 \mathrm{MHz}$, and $1.4 \mathrm{GHz}$. There are different considerations at each frequency and for each measurement including the degree of the thermal resolution, the beam size, and uncertainties.

For purposes of illustration, the results of their study at $1.4 \mathrm{GHz}$ based on the measurements of Klein et al. (1989) are shown in Figure 1. It is seen that the cosmic ray density level on the average is similar to our own on the average, although a bit lower if the assumptions stated at the beginning of this section are valid. Notice also that a nonuniform cosmic ray level is predicted.

When this figure is compared to that of the matter density distribution and the cosmic ray density, Fichtel, Ozel, and Stone (1990) conclude the two are compatible within an uncertainty of about 1.5 and a scale of coupling between the cosmic rays and metter of about $1 \mathrm{kpc}$ or greater. However, the scale of coupling is different on the two sides of the LMC.

\section{Summary}

The cosmic ray density in the LMC appears to be of the order of that in the local region of our own galaxy, but varying slowly with position. Further, a study of the synchrotron radiation and the matter column density in the LMC seems to indicate that it is possible to construct a consistent picture of the LMC based on the dynamic balance between the cosmic rays, the magnetic fields, and the kinetic motion of matter on the one side and gravitational attraction on the other and the additional feature that the magnetic field must be strong enough to contain the cosmic rays. A comparison of the contours related to the matter density and those 
related to the cosmic ray density predicted by the synchrotron radiation suggests that the scale of the coupling between the cosmic rays and the matter is probably of the order of a kiloparsec or larger since there is a smoothing required relative to the matter density controus to make the cosmic ray controus approximately consistent with those of the synchrotron radiation.

\section{References}

Cohen, R. S., T. M Dame, G. Garay, J. Montani, M. Rubio, and P. Thaddeus: 1988, Astroph. J. (Letters) 331, L95

Fichtel, C. E., Ozel, M. E., and Stone, R., 1990, Cosmic Rays and the Dynamic Balance in the Large Magellanic Cloud, submitted for publication.

Fisk, L. A.: 1971, J. Geophys. Res. 76, 221

Ginzburg, V. L. and Syrovatskii, S. I., 1964, The Origin of Cosmic Rays, New York: Macmillan.

Gleason, L. J. and Axford, W. I.: 1968, Astrophys. J. 154, 1011

Haynes, R. F., Klein, U., Wielebinski, R., Murray, J. D.: 1986, Astron. Astrophys. 159, 22

Klein, U., Wielebinski, R., Haynes, R. F. Malin, D. F.: 1989, Astron. Astrophys. 211, 280

Mathewson, D. J. and Ford, V. L., 1984, in Structure and Evolution of the Magellanic Clouds, eds.: S. van den Berg and K. S. de Boer, Dordrecht:Reidel, 125.

Ozel, M. E. and Fichtel, C. E.: 1988, Astroph. J. 335, 135

Parker, E. N.: 1965, Planet Space Sci. 13, 9

Simpson, J. A.: 1983, Ann. Rev. Nucl. Part. Sci. 33, 323-381

Webber, W. R., 1983, in Composition and Origin of Cosmic Rays, ed.: M. M. Shapiro, Dordrecht: Reidel, 83-100. 